\title{
The Role of Platelet-rich Plasma in Osteoarthritis of Knee-joint
}

Dr. Md. Nazibullah" ${ }^{1 *}$, Dr. Md. Tariqul Islam², Dr. Tapas Kumer Talukder ${ }^{3}$, Dr. A. K. M. Shaharul Islam ${ }^{4}$

\author{
${ }^{1}$ Associate Professor\& Head, Department of Orthopaedics \& Traumatology, TMSS Medical College, Bogura, Bangladesh \\ ${ }^{2}$ Assistant Professor, Department of Orthopaedics \& Traumatology, TMSS Medical College, Bogura, Bangladesh \\ ${ }^{3}$ Assistant Professor, Ortho Surgery, Shaheed Ziaur Rahman Medical College, Bogura, Bangladesh \\ ${ }^{4}$ Assistant Professor, Department of Orthopaedics \& Traumatology, TMSS Medical College, Bogura, Bangladesh
}

DOI: $10.36347 /$ sjams.2020.v08i08.003

| Received: 18.07.2020 | Accepted: 26.07.2020 | Published: 05.08.2020

*Corresponding author: Dr. Md. Nazibullah

Abstract

Original Research Article

Background: Osteoarthritis (OA) is a leading cause of disability and frequent visits to primary care practitioners for those with the condition in comparison to those without. OA affects the knee more often than any other joint of the human body. Platelet-rich plasma (PRP) which is also known as autologous conditioned plasma is a concentrate of platelet-rich plasma protein derived from whole blood, centrifuged to remove red blood cells. Now it is used in treating OA also. Aim of the study: The aim of this study was to evaluate the role of platelet-rich plasma in osteoarthritis of knee-joint. Materials and methods: This prospective observational study was conducted in the Department of Orthopaedics \& Traumatology, TMSS Medical College, Bogura, Bangladesh during the period from January 2018 to December 2018. In total 120 patients more than 40 years of age, diagnosed with grade I and II arthritis were included in this study as the study population. To all the participants three PRP injections were injected in the knees at 4-week interval. Patients were followed up and were assessed for pain and physical activity associated with arthritis using the Western Ontario and McMaster University Arthritis Index (WOMAC) score at the time of induction in the study and at 6 months interval. Results: The mean WOMAC score before the starting of treatment was 82.94 $( \pm 5.12)$ and after 6 months it was reduced to $37.87( \pm 4.73)$, which was statistically significant $(\mathrm{p}=0.0001)$. WOMAC score was improved more in the subgroup with patients having symptoms less than 2 years $(\mathrm{p}=0.0254)$. On the other hand, in case of subgroups with Kellgren-Lawrence grade 1 or 2 the p value was 0.0730 and there was not any significant difference in the WOMAC score at 6 months among males or females $(\mathrm{p}=0.1511)$. Conclusions: The proper sequential use of PRP injection for the treatment of osteoarthritis of knee-joint proved to be effective in our study. The findings of this study may be helpful in the treatment arena of osteoarthritis and in further similar researches.

Keywords: Platelet-rich plasma (PRP), Knee joint, Osteoarthritis.

Copyright @ 2020: This is an open-access article distributed under the terms of the Creative Commons Attribution license which permits unrestricted use, distribution, and reproduction in any medium for non-commercial use (NonCommercial, or CC-BY-NC) provided the original author and source are credited.

\section{INTRODUCTION}

Osteoarthritis (OA) is a leading cause of disability and doubles the number of visits to primary care practitioners for those with the condition in comparison to those without [1]. OA affects the knee more often than any other joint [2]. With the ageing of the population and the growing obesity epidemic, the number as well as the frequencies of surgical procedures for knee with OA will increase dramatically in the coming years. In medication knee replacement is the most costly to the health care system and burdensome for the patients also. OA is the most common articular disease that results from defects in articular cartilage. It affects significantly the quality of life and it is one of the main causes for musculoskeletal disability [3]. Osteoarthritis can affect any joint but mostly seen in knees, hip, hands, facet joints, and feet
[4]. With advancing age, there is increase in the prevalence of symptomatic knee osteoarthritis with the annual incidence being highest in people aged between 55 years and 64 years among the US population [5]. Osteoarthritis is not merely a process of cartilage wear and tear. Rather, it is a complex disease characterized by secretion of inflammatory mediators such as inflammatory cytokines which are claimed to play a leading role in the pathophysiology of this debilitating disease [6]. In a normal joint, generally chondrocytes remain inactive with very small turnover of the cartilage matrix. In osteoarthritis, stimulation of extra cellular matrix receptors over chondrocytes transforms them into activated chondrocytes. They exhibit cellular proliferation and cluster formation and produce matrix degrading proteinases and inflammatory cytokines [6]. In addition to cartilage destruction, osteoarthritis is also characterized by synovial inflammation, subchondral 
sclerosis, ligament laxity, and osteophyte formation [7]. The series of these pathological changes in all joint tissues led to the consideration of osteoarthritis as a disease of joint as an organ [6]. There have been a number of supportive treatments for osteoarthritis including analgesics, viscosupplements, and corticosteroids that are used to alleviate the symptoms of arthritis [8]. Recently, there is increase in the use of platelet-rich plasma (PRP) for the treatment of osteoarthritis. PRP derived from autologous blood containing growth factors is claimed to activate the healing process in damaged cartilage and helps in tissue regeneration [9]. Recent research has identified a number of key biochemical pathways that could be targeted therapeutically through biological intervention [10]. PRP is an autologous concentration of human platelets in a small volume of plasma, where the platelet concentration is higher (Typically up to five times higher) than the normal platelet concentration in a healthy person's blood. Emerging evidence suggests PRP has the potential to have a regenerative effect on certain body tissues, in addition to the main role platelets play in haemostasis [11]. PRP has been shown to provide some symptomatic relief in early $\mathrm{OA}$ of the knee and to be at least as effective as intra- articular hyaluronic acid and steroid injections for symptom control [12]. This therapy is a minimally invasive intervention which could be used to enhance tissue regeneration. PRP contains alpha granules, in which about $70 \%$ of their growth factors will be secreted in the first $10 \mathrm{~min}$, and almost all the stored amount will be released in the first hour [13].

\section{Methodology and Materials}

This prospective observational study was conducted in the Department of Orthopaedics \& Traumatology, TMSS Medical College, Bogura, Bangladesh during the period from January 2018 to December 2018. In total 120 patients within 40 to 60 years of age diagnosed with grade I and II arthritis were included in this study as the study population. To all the participants three PRP injections were injected in the knees at 4-week interval. Patients were followed up and were assessed for pain and physical activity associated with arthritis using the Western Ontario and McMaster University Arthritis Index (WOMAC) score at the time of induction in the study and at 6 months interval. All patients were diagnosed with grade I and II (KellgrenLawrence [14]) osteoarthritis, symptoms and Western Ontario and McMaster University Arthritis Index (WOMAC) score [15]. Dhurat and Sukesh[16] described two methods for preparation of PRP using differential centrifugation, namely PRP method and buffy-coat method. We utilized PRP method in our study group. Patients were registered with the Orthopedic Department of mentioned hospital and PRP was prepared at Hematology Department of the same hospital. Blood was drawn from patient's cubital vein to make $4-6 \mathrm{~mL}$ of PRP. Blood samples were collected in bags containing anticoagulant (citrate phosphate dextrose and adenine). The collected blood samples were then transferred into a tube and centrifuged at a constant acceleration to separate the red blood cells (RBCs) from whole blood volume. It separates blood in three layers (platelets with white blood cells (WBCs), buffy coat rich in WBCs, and RBCs from top to bottom). Then, the upper layer and superficial buffy coat are centrifuged again in a new sterile tube sufficient enough to form soft pellets at the bottom. Upper two thirds of this centrifuged volume is discarded, while the remaining lower one third is homogenized to make PRP. The PRP was dispensed in a sterile syringe. Patients were given appointment in Outpatient Department (OPD) for PRP injection. Under aseptic technique, PRP was injected in the knees through supra-lateral approach in supra-patellar pouch. After given with injection, patients were observed for $20 \mathrm{~min}$ and discharged home with clear instructions for signs of infection including increasing pain, redness, warmth, and inability to bear weight. Patients were prescribed paracetamol in case of pain and advised not to take non-steroidal anti-inflammatory drugs (NSAIDs) and steroids. Patients were followed up in OPD on regular basis. A total of three injections were given to each patient at 4 weeks interval. WOMAC score was calculated at the time of induction in the study and at 6 months interval to assess the effect of PRP injection. Data were presented as frequencies. WOMAC score at the start of treatment was compared with the final scores.

\section{RESULTS}

In this study the total participants were 120 in number who had completed the full tenure of this intervention. The mean age of the patients was 54.76 $(+7.02)$ years. In analyzing the gender of the participants of this study we found that, among 120 participants, $27 \%(n=33)$ were male and $73 \%(n=87)$ were female. So male were dominating in number and the male-female ratio was 1:2.64. Among total participants in total $86(71.67 \%)$ were classified as having Kellgren-Lawrence grade 1 osteoarthritis, while $34(28.33 \%)$ were having Kellgren-Lawrence grade 2 osteoarthritis. In this study we found, most of the patients $(n=105 ; 87.5 \%)$ had clinical symptoms for less than 2 years and $15(12.5 \%)$ for more than 2 years. The mean WOMAC score before the starting of treatment was $82.94( \pm 5.12)$ and after 6 months it was reduced to $37.87( \pm 4.73)$, which was statistically significant $(\mathrm{p}=$ $0.0001)$. WOMAC score was improved more in the subgroup with patients having symptoms less than 2 years $(p=0.0254)$. On the other hand, in case of subgroups with Kellgren-Lawrence grade 1 or 2 the $p$ value was 0.0730 and there was not any significant difference in the WOMAC score at 6 months among males or females $(\mathrm{p}=0.1511)$. In total two patients developed minor hyperemia which was managed conservatively with activity limitation and ice application, but none of the patient developed gross infection. 


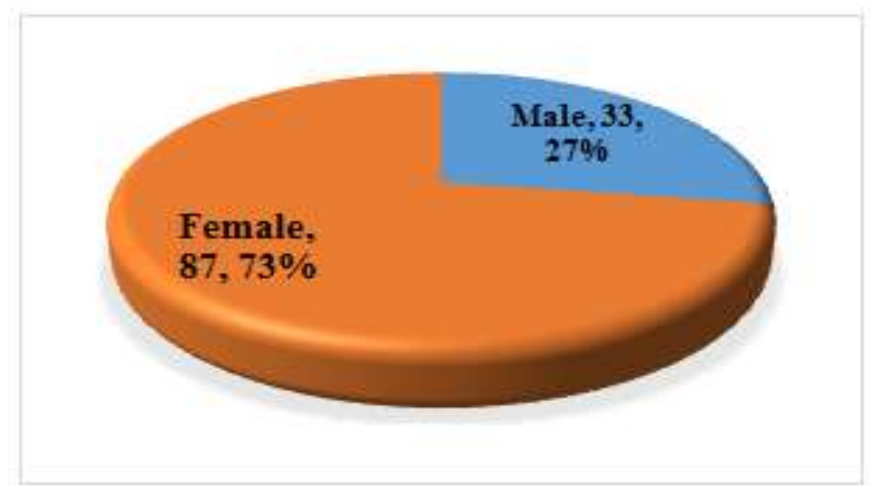

Fig-I: Gender distribution of participants $(\mathrm{N}=120)$

Table-I: Difference between WOMAC score at the start of treatment and at 6 months follow-up $(\mathrm{N}=120)$

\begin{tabular}{|l|c|c|c|}
\hline Period & Mean & SD & P value \\
\hline At the start of treatment & 82.94 & 5.12 & \multirow{2}{*}{0.0001} \\
\cline { 1 - 3 } At 6 months follow-up & 37.87 & 4.73 & \\
\hline
\end{tabular}

Tale-II: Comparison of WOMAC score after 6 months between subgroups $(\mathrm{N}=120)$

\begin{tabular}{|c|c|c|c|}
\hline Component & Mean & SD & P value \\
\hline \multicolumn{4}{|c|}{ Gender } \\
\hline Male & 37.71 & 5.82 & \multirow[t]{2}{*}{0.1511} \\
\hline Female & 39.32 & 5.45 & \\
\hline \multicolumn{4}{|c|}{ Kellgren-Lawrence grade } \\
\hline Grade-1 & 37.88 & 5.78 & \multirow[t]{2}{*}{0.0730} \\
\hline Grade-2 & 39.94 & 5.19 & \\
\hline \multicolumn{4}{|c|}{ Duration of symptoms } \\
\hline$\leq 2$ Years & 38.39 & 5.46 & \multirow[t]{2}{*}{0.0254} \\
\hline$>2$ Years & 41.73 & 4.38 & \\
\hline
\end{tabular}

\section{DISCUSSION}

In this current study, we observed remarkable clinical improvement among patients with arthritis who received PRP injection. Difference in WOMAC score at 6 months interval was significant. Patients having symptoms for less than 2 years showed more improvement in WOMAC score. Unfortunately, in our study, we did not have any comparative group with other mode of treatment. But in the literature, there are some comparative studies and meta-analyses favoring the use of osteoarthritis are a debilitating disease affecting the mobility and quality of life among individuals. Nonsurgical treatments including PRP, corticosteroids, and hyaluronic acid have been prescribed by physicians with good short-term results. There has been increasing interest in biological and regenerative methods including PRP [17]. However, it is to be noted that any invasive procedure may have some placebo effect. To better understand the effect of each treatment modality, it is always recommended to have a alities with favorable outcomes. A recent systematic review and meta-analysis by Sadabad et al. showed superior efficacy for PRP as compared to hyaluronic acid in alleviating the clinical symptoms over a period of 2 years. However, they recommended the need of long- term studies for the effects of PRP [12]. Another systematic review by Khoshbin et al. shows that multiple sequential PRP injections have better symptomatic relief as compared to hyaluronic acid or normal saline solution at 6 months follow- up [18]. In our study also we found significant improvement in the WOMAC score in patients with PRP injections at 6 months interval. PRP therapy is minimally invasive and a simple alternative, being used to enhance the healing process and tissue regeneration. It has the potential to improve the quality of life, relieve pain, and enhance knee joint function [19]. Kon et al. studied the effects of PRP injection in the treatment of chronic degenerative condition of the knees. They studied the effects in 115 patients at 6 and 12 months follow-up. There was an overall significant improvement at 6 and 12 months interval. However, they observed that in the initial 6 months, the results were stable; in the second half (7-12 months), the results became worse at 12 months as compared to the first 6 months. In addition, the results were better in patients with early grade of arthritis [20]. In our study, we had favorable results at 6 months, but our overall study duration was shorter (i.e. 6 months follow-up only). Secondly, we had selected patients with early arthritis (Kellgren grade 1 and 2). Jang et al. presented the outcome of his 65 patients who were treated with PRP injection. There was improvement in Visual analogue scale (VAS) and International Knee Documentation Committee (IKDC) scores; however, the developing degeneration in the joint had been associated with reduced clinical effects of PRP. The 
results were better in younger age-group with early osteoarthritis [21]. In our study, the study group was relatively younger with early arthritis and showed significant improvements at 6 months follow-up. Patel et al. compared the effects of PRP in three sub-groups and concluded that there was a significant improvesment in patients receiving either single (group A) or two (group B) PRP injections. The third group (group C), received normal saline instead of PRP, showed deterioration of symptoms. Mild complications including nausea and dizziness were observed in some patients but for shorter duration [22]. In our study, unfortunately, there was no comparison group, but PRP injection showed significant clinical improvement. Mild hyperemia was observed in three patients but managed conservatively.

\section{Limitations OF THE STUdy}

This was a single centered study with a small sized sample. So the findings of this study may not reflect the exact scenario of the whole country.

\section{CONCLUSION AND \\ RECOMMENDATIONS}

The proper sequential use of PRP injection for the treatment of osteoarthritis of knee-joint proved to be effective in our study. The findings of this study may be helpful in the treatment arena of osteoarthritis and in further similar researches. For getting more specific findings we would like to recommend for conducting similar more studies in several places.

\section{REFERENCES}

1. Wright EA, Katz JN, Cisternas MG, Kessler CL, Wagenseller A, Losina E. Impact of knee osteoarthritis on health care resource utilization in a US population-based national sample. Med Care. 2010;48(9):785-91.

2. Duymus TM, Mutlu S, Dernek B, Komur B, Aydogmus S, Kesiktas FN. Choice of intra-articular injection in treatment of knee osteoarthritis: plateletrich plasma, hyaluronic acid or ozone options. Knee Surg Sports Traumatol Arthrosc. 2017;25(2):48592.

3. Raeissadat SA, Rayegani SM, Babaee M, Ghorbani E. The effect of platelet-rich plasma on pain, function, and quality of life of patients with knee osteoarthritis. Pain Research and Treatment. 2013 Jan 1;2013.

4. Litwic A, Edwards MH, Dennison EM, Cooper C. Epidemiology and burden of osteoarthritis. British medical bulletin. 2013 Mar 1;105(1):185-99.

5. Deshpande BR, Katz JN, Solomon DH, Yelin EH, Hunter DJ, Messier SP, Suter LG, Losina E. Number of persons with symptomatic knee osteoarthritis in the US: impact of race and ethnicity, age, sex, and obesity. Arthritis care \& research. 2016 Dec;68(12):1743-50.
6. Kapoor M, Martel-Pelletier J, Lajeunesse D, Pelletier JP, Fahmi H. Role of proinflammatory cytokines in the pathophysiology of osteoarthritis. Nature Reviews Rheumatology. 2011 Jan;7(1):33.

7. Martel-Pelletier J, Boileau C, Pelletier JP, Roughley PJ. Cartilage in normal and osteoarthritis conditions. Best practice \& research Clinical rheumatology. 2008 Apr 1;22(2):351-84.

8. Michael JW, Schlüter-Brust KU, Eysel P. The epidemiology, etiology, diagnosis, and treatment of osteoarthritis of the knee. Deutsches Arzteblatt International. 2010 Mar;107(9):152.

9. Smyth NA, Haleem AM, Ross KA, Hannon CP, Murawski CD, Do HT, Kennedy JG. Platelet-rich plasma may improve osteochondral donor site healing in a rabbit model. Cartilage. 2016 Jan;7(1):104-11.

10. Patel S, Dhillon MS, Aggarwal S, Marwaha N, Jain A. Treatment with platelet- rich plasma is more effective than placebo for knee osteoarthritis: a prospective, double-blind, randomized trial. Am J Sports Med. 2013;41(2):356-64.

11. Lee KS, editor. Platelet-rich plasma injection. Seminars in musculoskeletal radiology. New York: Thieme Medical Publishers; 2013.

12. Lana JF, Weglein A, Sampson SE, Vicente EF, Huber SC, Souza CV, Ambach MA, Vincent H, Urban-Paffaro A, Onodera CM, AnnichinoBizzacchi JM. Randomized controlled trial comparing hyaluronic acid, platelet-rich plasma and the combination of both in the treatment of mild and moderate osteoarthritis of the knee. Journal of stem cells \& regenerative medicine. 2016;12(2):69.

13. Spakova T, Rosocha J, Lacko M, Harvanova D, Gharaibeh A. Treatment of knee joint osteoarthritis with autologous platelet-rich plasma in comparison with hyaluronic acid. Am J Phys Med Rehabil. 2012;91(5):411-7.

14. Kellgren JH and Lawrence JS. Radiological assessment of osteo-arthrosis. Ann Rheum Dis. 1957; 16(4): 494-502.

15. McConnell S, Kolopack P, and Davis AM. The Western Ontario and McMaster Universities Osteoarthritis Index (WOMAC): a review of its utility and measurement proper- ties. Arthritis Rheum. 2001; 45(5): 453-461.

16. Dhurat R and Sukesh M. Principles and methods of preparation of platelet-rich plasma: a review and author's perspective. J Cutan Aesthet Surg. 2014; 7(4): 189-197.

17. Andia I, Sánchez M, Maffulli N. Joint pathology and platelet-rich plasma therapies. Expert opinion on biological therapy. 2012 Jan 1;12(1):7-22.

18. Khoshbin A, Leroux T, Wasserstein D, Marks P, Theodoropoulos J, Ogilvie-Harris D, Gandhi R, Takhar K, Lum G, Chahal J. The efficacy of platelet-rich plasma in the treatment of symptomatic knee osteoarthritis: a systematic review with quantitative synthesis. Arthroscopy: The Journal of 
Arthroscopic \& Related Surgery. 2013 Dec 1;29(12):2037-48.

19. Filardo G, Kon E, Buda R, Timoncini A, Di Martino A, Cenacchi A, Fornasari PM, Giannini S, Marcacci M. Platelet-rich plasma intra-articular knee injections for the treatment of degenerative cartilage lesions and osteoarthritis. Knee Surgery, Sports Traumatology, Arthroscopy. 2011 Apr 1;19(4):52835.

20. Kon E, Buda R, Filardo G, Di Martino A, Timoncini A, Cenacchi A, Fornasari PM, Giannini S, Marcacci M. Platelet-rich plasma: intra-articular knee injections produced favorable results on degenerative cartilage lesions. Knee Surgery, Sports Traumatology, Arthroscopy. 2010 Apr 1;18(4):4729.

21. Jang SJ, Kim JD, and Cha SS. Platelet-rich plasma (PRP) injections as an effective treatment for early osteoarthritis. Eur J Orthop Surg Traumatol. 2013; 23(5): 573-580.

22. Patel S, Dhillon MS, Aggarwal S, Marwaha N, Jain A. Treatment with platelet-rich plasma is more effective than placebo for knee osteoarthritis: a prospective, double-blind, randomized trial. The American journal of sports medicine. 2013 Feb;41(2):356-64. 